\title{
LES METASTASES MENINGEES SOLITAIRES PREVALENTES
}

\section{SOLITARY LEPTOMENINGEAL METASTASES}

EL ASRI Abad Cherif ${ }^{1}$

GAZZAZ Miloudi ${ }^{1}$

AKHADDAR Ali ${ }^{1}$

NAAMA Okacha ${ }^{1}$

BOULHROUD Omar ${ }^{1}$

BELHACHMI Adil ${ }^{1}$

BELFKIH Hatim ${ }^{1}$

EL MOSTARCHID Brahim ${ }^{1}$

BOUCETTA Mohammed ${ }^{1}$

1. Services de Neurochirurgie, hôpital militaire Mohammed V, CHU Rabat, Maroc

E-Mail Contact - EL ASRI Abad Cherif : abad20031 (at) hotmail (dot) com

\begin{tabular}{l}
\hline RESUME \\
Objectif \\
Intérêt d'avoir une confirmation histologique, en présence d'un processus leptoméningée solitaire dont le \\
diagnostic de méningiome bénin est souvent évoqué en premier. \\
Introduction \\
Les métastases méningées sont observées de plus en plus fréquemment chez les patients connus porteur \\
d'une néoplasie, du fait de l'allongement de la survie des patients et l'amélioration des moyens \\
diagnostiques; elles représentent environ $8 \%$ des métastases du système nerveux central. \\
Observation \\
Nous rapportons deux observations originales de patients sans histoire néoplasique, opérés pour un \\
processus leptomeningé solitaire dont le diagnostic préopératoire était celui d'un méningiome. L'étude \\
histologique révélait la nature néoplasique métastatique de la lésion, alors que le bilan radiologique a \\
permis de détecter la localisation primitive méconnue. \\
Conclusion et discussion \\
La découverte à l'occasion d'une imagerie cérébrale (TDM et/ou IRM) chez un patient, sans histoire \\
néoplasique, d'une ou plusieurs lésions leptoméningées, pose un problème diagnostique. Lorsque la \\
localisation est unique, le diagnostic de méningiome est évoqué en premier; alors que les lésions \\
inflammatoires et secondaires des hémopathies malignes représentent un diagnostic différentiel lorsque les \\
lésions sont diffuses. Seront discutées à lumière de ces observations et d'une revue de la littérature, les \\
aspects physiopathologiques, cliniques, paracliniques, thérapeutiques et évolutifs de cette pathologie.
\end{tabular}




\section{SUMMARY}

\section{Background and purpose}

The need of histological confirmation for solitaries meningeal process witch are often misdiagnosed as benign meningiomas.

\section{Introduction}

Leptomeningeal metastases are more frequently observed in patient with known neoplasm, because cancer patients are living longer and advance in diagnostic means. They represent approximately $8 \%$ of metastases of the central nervous system, but rarely diagnosed in the absence of diagnosed primary tumour.

\section{Observation}

we report tow cases of patients without neoplasic history, which were operated for presumed intracranial meningioma and the histopathology of specimen demonstrated that it was a meningeal metastases. The misdiagnosed primary location was detected by the body imaging.

\section{Discussion and conclusion}

The presence of solitary or multiple leptoméningeal lesions in cranial imaging (CT-scan or MRI) of patient without neoplasic history is often misdiagnosed. For the isolated dural-based metastasis, differentiation from meningioma by conventional imaging may be difficult. In multiples lesions, inflammatory or secondary homeopathy, are evocated as probable diagnoses. In light of those observations and reviewing the literature, the pathophysiological, clinical, paraclinic, therapeutic aspects and evolution will be discussed.

\section{INTRODUCTION}

La dissémination intracrânienne des cellules tumorales métastatiques reste une complication redoutable qui survient généralement au stade tardif de l'évolution de la maladie cancéreuse. Les études autopsiques montrait que 20 à $25 \%$ des patients souffrant d'un cancer développeraient des métastases cérébrales(13). Si les localisations cérébrales ou médullaires sont bien connues et sont facilement diagnostiquées grâce au développement des moyens d'imagerie ; les localisations méningées isolées (sans lésions cérébrales concomitantes) que se soit unique ou diffuses sont plus rares et leur incidence a été rarement étudiée. Cette localisation méningée est souvent diffuse réalisant une véritable carcinomatose méningée ou plus rarement unique et prenant l'aspect d'un processus leptomeningé avec attache dural. Cependant, La découverte à l'occasion d'une imagerie cérébrale (TDM et/ou IRM) chez un patient, sans histoire néoplasique, d'une ou plusieurs lésions leptoméningées, pose un problème diagnostique. Lorsque la localisation est unique, le diagnostic de méningiome est évoqué en premier ; alors que les lésions inflammatoires et secondaires des hémopathies malignes représentent un diagnostic différentiel lorsque les lésions sont diffuses(8, 12). Exceptionnellement les tumeurs primitives du SNC sont à l'origine des lésions leptomeningé par dissémination des cellules tumorales. Nous rapportons deux observations originales de patients sans histoire néoplasique, opérés pour un processus leptomeningé métastatique dont le diagnostic préopératoire était celui d'un méningiome.

\section{CAS CLINIQUES}

\section{Observation $\mathrm{N}^{\circ} 1$}

Un patient âgé de 40 ans, sans antécédents pathologiques, a été admis au service de neurochirurgie pour l'installation rapidement progressive depuis 15 jours d'une hémiparésie droite. Aucun signe urinaire ou abdominal n'a été retrouvé à l'interrogatoire et à l'examen clinique. L'IRM cérébrale montrait un processus intracrânien fronto-parietal, bien limité et se rehaussant d'une manière homogène après l'injection du gadolinium (figure 1). L'aspect radiologique de ce processus présentant une base d'insertion leptoméningée évoquait un méningiome. La résection totale de la tumeur a été réalisée à travers une craniectomie frontoparietale. L'examen histologique et immuno-histochimique de la pièce opératoire montrait qu'il s'agissait d'une localisation secondaire d'un adénocarcinome rénal. La TDM thoracoabdominale réalisée, montrait l'existence d'une masse rénale gauche d'allure tumorale, étendue à la graisse peri-rénale sans autres localisations secondaires associées. Une néphrectomie gauche large a été réalisée dans l'immédiat puis un complément de radiothérapie cérébrale externe a été préconisé. L'évolution clinique a été marquée par une récupération complète du déficit neurologique. Le suivie du patient pendant huit mois n'a pas montré de signes de récidives locales ou à distance.

\section{Observation $\mathrm{N}^{\circ} 2$}


Un patient de 65 ans, tabagique chronique, a été admis dans notre formation pour l'apparition progressive depuis 2 mois de vertiges, céphalées et de vomissement. L'examen neurologique trouvait un syndrome cérébelleux kinétique associés à des signes d'irritation pyramidale du coté gauche. Le reste de l'examen somatique était normale. Une TDM cérébrale montrait un processus de la fosse cérébrale postérieure homogène se rehaussant fortement après contracte responsable d'une dilatation ventriculaire. L'IRM cérébrale montrait qu'il s'agissait d'un processus de l'angle ponto-cerebelleux gauche avec base d'implantation sur la tente du cervelet prenant le gadolinium d'une manière homogène ; Evoquant un méningiome de la tente du cervelet (figure 2). Le patient fut opéré par voie rétrosigmoïdienne gauche avec réalisation d'une exérèse complète du processus extra-axial à base d'insertion dural. Les suites opératoires furent marquées par une amélioration de la symptomatologie alors que l'examen histologique montrait q'il s'agit d'un métastase d'un adénocarcinome pulmonaire. Un processus pulmonaire de siège hilaire gauche d'allure tumoral a été mis en évidence par la TDM thoracique et dont la biopsie sous fibroscopie confirmait la nature carcinomateuse de la lésion. Une chimiothérapie systémique fut préconisée en post-opératoire, puis le patient a été perdu de vu.

\section{DISCUSSION}

Chez les patients traités pour une maladie néoplasique systémique, La localisation intracrânienne constitue un abri des cellules tumorales à l'action des traitements anticancéreux dont la plupart ne traverse pas la barrière hémato-encéphalique. Les métastases méningées, observées de plus en plus fréquemment du fait de l'allongement de la survie des patients et du développement des moyens diagnostiques d'imagerie et cytologiques, représentent environ $8 \%$ des métastases du SNC, les localisations leptoméningées étant plus fréquentes que les localisations durales(10). Cependant, L'incidence des lésions méningées métastatiques n'est pas bien définie, et la plupart des séries rapportent des études autopsiques. Les lésions métastatiques leptoméningées peuvent être secondaires à des tumeurs extracraniennes solides, des cancers hématologiques ou parfois des tumeurs primitifs du système nerveux central. L'étude autopsique réalisée par Posner and Chernik sur 2375 cas de patients présentant un cancer systémique retrouvait $9 \%$ de localisations léptoméningées, dont seulement $4 \%$ ne sont pas associées à d'autres lésions cérébrales(8). D'autres séries rapportaient une incidence variable entre 5 et $10 \%(8,12,13)$. Toutes les tumeurs peuvent métastaser aux leptoméninges mais surtout les cancers du sein, bronchique, digestif, les lymphome et le mélanome ont une grande affinité pour les méninges ; d'autres sont exceptionnellement retrouvés dans cette localisation tel que le cancer du rein, l'ovaire et les carcinomes thyroïdiens $(8,12,13)$. Le diagnostic des lésions métastatiques méningées se fait au stade tardif de l'évolution de la maladie cancéreuse ou bien souvent en post-motum. Rarement la localisation méningée est révélatrice du néoplasme primitif, ceci a été retrouvé dans $1 \%$ des cas. C'est dans cette dernière situation que le diagnostic d'une métastase est rarement posé(12).

Cependant, Plusieurs théories viennent expliquer la localisation leptoméningée des cellules tumorales. L'extension par contiguïté à partir d'une métastase cérébrale corticale, paraventriculaire ou par envahissement durale à partir d'une métastase osseuse de la voûte ou de la base du crâne constitue l'une des possibilités d'extension tumorale de plusieurs tumeurs primitives à savoir les carcinomes prostatiques, pulmonaires et les mélanomes(8). Les cellules tumorales peuvent emprunter la circulation artérielle et atteindre le plexus choroïde, puis disséminer à travers le liquide céphalo-rachidien vers les leptomeninges. L'atteinte méningée peut se faire directement à partir des artères méningées ; ceci a été documenté par quelques auteurs en montrant une vascularisation tumorale par le biais des vaisseaux méningées sur les artériographies réalisées(12). La voie veineuse à travers le plexus veineux paravértbrale de Batson peut être la principale voie de dissémination des cellules tumorale vers le liquide céphalo-rachidien et vers les méninges(8). Les localisations méningées métastatiques peuvent survenir après une résection chirurgicale d'un processus tumoral cérébral primitif ou secondaire, ceci par dissémination des cellules tumorales dans les espaces sous arachnoïdienne ; ce mécanisme iatrogène est surtout incriminer dans la dissémination méningée des gliomes malins, les médulloblastomes, les ependymomes et dans les métastases siégeant au niveau de la fosse cérébrale postérieure (16).

Cette localisation leptomeningée peut être le premier et le seul point d'appel d'une tumeur non encor diagnostiquée ; elle se manifeste cliniquement par les signes compressifs et irritatifs du parenchyme adjacent et qui vient mimer la symptomatologie des méningiomes. L'infiltration leptomeningée diffuse se déclare par des signes de méningisme, d'atteinte multiple des nerfs crâniens, des troubles de la conscience ; elle est souvent plurisymptomatique et d'installation rapide. Les signes neurologiques uniques et isolés peuvent être vu dans 30 à $53 \%$ des cas (15). Ces signes cliniques sont en rapport avec obstruction des voies d'écoulement de LCR, irritation méningée, irritation voir envahissement du parenchyme cérébral adjacent ou 
des nerfs crâniens ou rachidiens. Des lésions cérébrales ou médullaires ischémiques peuvent être secondaire à une atteinte vasculaire associée avec perturbation du métabolisme cérébral $(1,9)$.

Si le diagnostic des carcinomatose méningée néoplasique reposait sur l'apport de l'étude cytologique de LCR et sur les études autopsiques ; récemment l'imagerie cranio-spinale permet un diagnostic précoce de plus en plus sensible et spécifique grâce au développement des acquisitions et des séquences IRM. D'autre part l'aspect radiologique des métastases leptoméningées solitaires est difficile à distinguer des autres processus méningée classiques ; avec en premier les méningiomes ; ces métastases paraissent en iso signal T1 et T2 avec un rehaussement intense et homogène au produit de contraste à base d'insertion dural et présentant le signe classique de la queue de comète décrit comme signe pathognomonique des méningiomes(12). D'autres néoplasmes à savoir le palsmocytome, le lymphome, l'ependymome, l'actinomycome et le xanthoastrocytome ou granulomatoses tel que le tuberculome, la sarcoïdose et la pachyméningite peuvent prendre le même aspect radiologique $(7,10)$.

La sensibilité du scanner après injection du PC est relativement faible, elle révèle des anomalies dans seulement 25 à $50 \%$ à type de rehaussement méningée, obstruction de citernes de la base et ou hydrocéphalie. L'IRM est plus sensible que la TDM dans la détection des métastases leptoméningées, en effet la Sp T1 et récemment les séquences FLAIR avec injection du gadolinium sont les examens de choix. L'IRM montre les mêmes anomalies détectées à la TDM mais avec une meilleure sensibilité et spécificité. Les lésions peuvent prendre un aspect curviligne ou nodulaire des leptomeninges des citernes de la base et ou des sillons corticaux dans $35 \%$ des cas; I'hydrocéphalie est vue dans $13 \%$ des cas, et l'infiltration des nerfs crâniens se voit dans $11 \%$ des cas. La co-existence d'autres lésions cérébrales estvue dans $17 \%$ des $\operatorname{cas}(2,3)$. Rarement les métastases sont découvert à l'occasion d'un hématome sous dural chronique récidivant qui pourrait masquer la lésion leptomeningée secondaire(14).

L'étude cytologique du LCR constitue le gold standard dans le diagnostic des métastases leptoméningées. Elle peut montrer des perturbations biochimiques ou cytologiques à type d'hypoglucorrachie dans $30 \%$ des cas, hyperproteinorrachie dans $80 \%$ des cas, une augmentation des taux des leucocytes surtout des lymphocytes, mais surtout la présence des cellules tumorales. La sensibilité de cet examen peut atteindre $85 \%$ après une deuxième voire un troisième prélèvement de LCR $(5,11)$.

Sur le plan thérapeutique, ces lésions lorsqu'elles sont solitaires, et à cause de leurs aspects imagerie et en dehors d'un contexte néoplasique, sont souvent opérées à tort pour des méningiomes bénins. Comme c'était le cas chez nos deux patients et dans la plupart des cas rapportés, l'exérèse complète de cette tumeur, dont l'aspect macroscopique est celui d'un méningiome avec un plan arachnoïdien de clivage par rapport au parenchyme adjacent, est facile ; et seul l'examen histologique qui permet de redresser le diagnostic. Ainsi fait ; une recherche de la localisation primitif et d'autres localisations métastatiques seraient réalisées.

En effet la conduite thérapeutique ultérieure constitue un sujet de controverse, faut il s'acharner à proposer un traitement agressif chez ces patients ? Cependant, la localisation méningée est reconnue comme un facteur péjoratif de l'évolution d'une maladie cancéreuse, avec une survie moyenne ne dépassant pas les quelques semaines à quelques mois (6). Chez les patients qui n'ont pas de maladie systémique évolutive associée, la moyenne de survie est de 8 mois et le taux de survie à 1 an est de $22 \%$; ceci s'était le cas chez notre premier patient, ou la résection chirurgicale de la lésion primitive fut réalisée en complément. Le traitement par radiothérapie et chimiothérapie intrathécale permet une amélioration ou une stabilisation des symptômes neurologiques dans 30 à $70 \%$ des cas selon les séries(4). Cependant, malgré l'amélioration initiale, la moyenne de survie des patients traités n'est que de 3 ou 4 mois tous cancers confondus et le taux de survie à 1 an dépasse rarement $10 \%$. En effet, le choix de la thérapeutique serait fonction de la nature de la tumeur, de son potentiel évolutif, du statut neurologique et le Karnofsky du patient. Et par conséquent, il faut décider s'il y a lieu d'entreprendre un traitement énergique avec un faible espoir d'amélioration prolongée, ou s'il faut se contenter de mesures purement symptomatiques à visée antalgique $(4,6,11)$. 

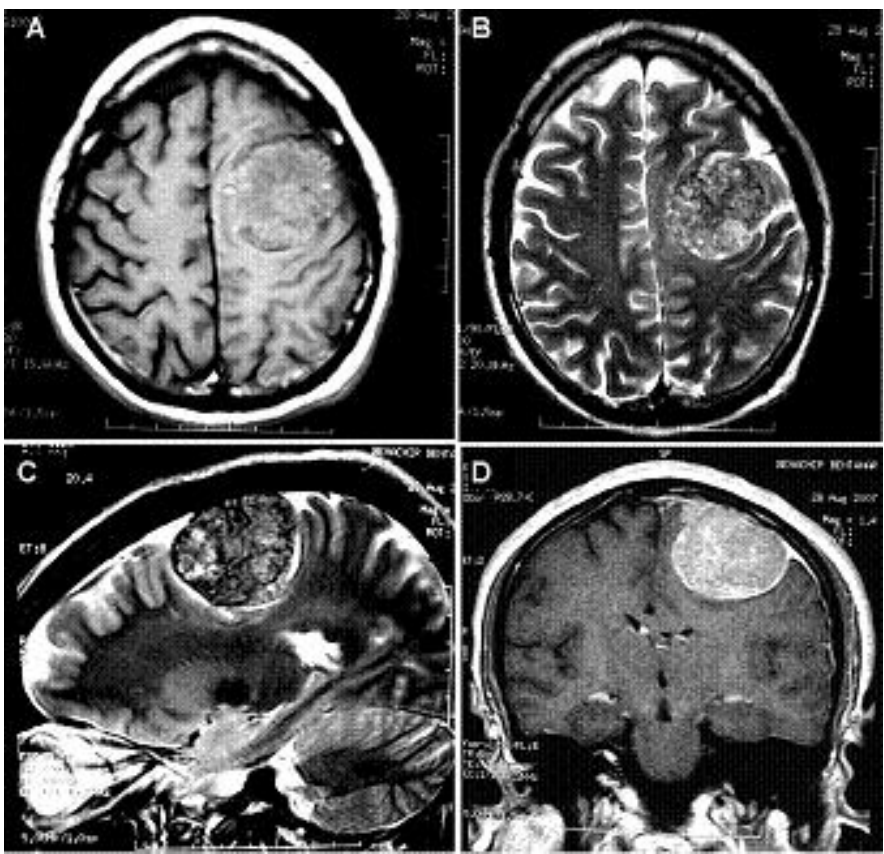

Figure 1

IRM cérébrale en Sp T1 avant injection du gadolinium (A) et après injection (D) et en Sp T2 (B et D) montrant un processus fronto-rolondique gauche en iso signal T1 et T2 se rehaussant après injection du gadolinium et base d'insertion durale évoquant un méningiome de la convexité.

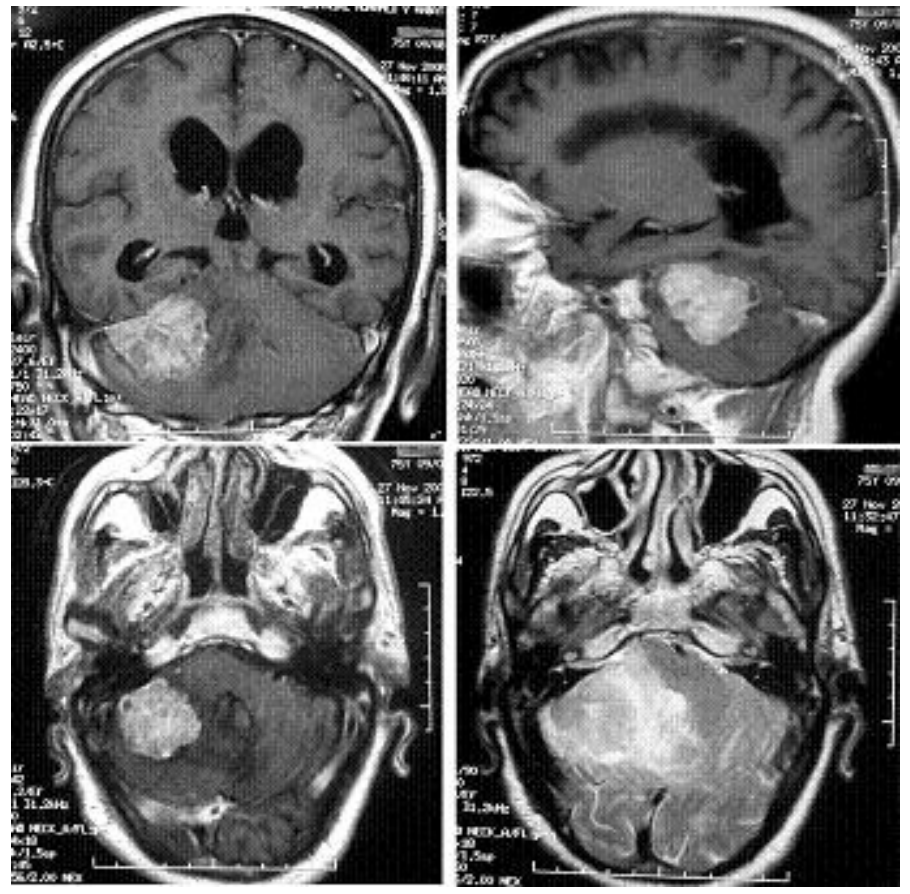

Figure 2

IRM cérébrale avec acquisitions dans les trois plans de l'espace et en Sp T1 après injection du Gadolinium et en Sp T2 montrant le processus de la fosse cérébrale postérieure à base d'insertion sur la tente du cervelet, et on note l'oedème périlésionnel intéressant le tronc cérébral. 


\section{REFERENCES}

1. CASTRO MP, MCDONALD TJ, QUALMAN SJ, ODORISIO TM. Cerebrospinal fluid gastrin releasing peptide in the diagnosis of leptomeningeal metastases from small cell carcinoma. Cancer 2001; 91:2122-6.

1. CHAMBERLAIN MC, KORMANIK PA: Prognostic significance of coexistent bulky metastatic central nervous system disease in patients with leptomeningeal metastases. Arch Neurol 1997; 54:13641368.

2. COLLIE DA, BRUSH JP, LAMMIE GA, GRANT R, KUNKLER I, LEONARD R, GREGOR A, SELLAR RJ: Imaging features of leptomeningeal metastases. Clin Radiol 1999; 54:765-771.

3. DEANGELIS LM, BOUTROS D. Leptomeningeal metastasis. Cancer Invest 2005;23(2): 145-54.

4. FREILICH RJ, KROL G, DEANGELIS LM: Neuroimaging and cerebrospinal fluid cytology in the diagnosis of leptomeningeal metastasis. Ann Neurol 1995; 38:51-5,.

5. HERRLINGER U, FORSCHLER H, KUKER W, ET AL. Leptomeningeal metastasis: survival and prognostic factors in 155 patients. J Neurol Sci 2004;223: 167-178.

6. LAIDLAW JD, KUMAR A, CHAN A. Dural metastases mimicking meningioma. Case report and review of the literature. J Clin Neurosc; 2004;11:780-3.

7. LAIGLE-DONADEY F, TAILLIBERT S, MOKHTARI K, HILDEBRAND J, DELATTRE JY. Dural metastases. J Neurooncol 2005;75: 57-61.

8. MEHTA BM, GLASS JP, SHAPIRO WR. Serum and cerebrospinal fluid distribution of 5methyltetrahydrofolate after intravenous calcium leucovorin and intra-Ommaya methotrexate administration in patients with meningeal carcinomatosis. Cancer Res 1983; 43:435-8.

9. NAGGARA O, BRAMI-ZYLBERBERG F, RODRIGO S, RAYNAL M, MEARY E, GODON-HARDY S, OPPENHEIM C, MEDER JF. Imagerie des métastases intracrâniennes chez l'adulte. J Radiol 2006;87:792-806.

10.POSNER JB. Neurologic Complications of Cancer. Philadelphia, PA: F.A. Davis Company, 1995.

11. TAGLE P, VILLANUEVA P, TORREALBA G, HUETE I. Intracranial metastasis or meningioma? An uncommon clinical dilemma. Surg Neurol 2002; 58: 241-5.

12.TAKAKURA K, SANO K, HOJO S. Metastatic tumors of the central nervous system. Tokyo : Igaku Shoin, 1982.

13.TSENG SH, LIAO CC, LIN SM, CHEN Y, SHUN CT: Dural metastasis in patients with malignant neoplasm and chronic subdural hematoma. Acta Neurol Scand 2003; 108:43-46.

14.TWIJNSTRA A, VAN ZANTEN AP, NOOYEN WJ, ONGERBOER DE VISSER BW. Sensitivity and specificity of single and combined tumour markers in the diagnoses of leptomeningeal metastases from breast cancer. J Neurol Neurosurg Psychiatry 1986: 49; 1246-50.

15.VAN DER REE TC, DIPPEL DW, AVEZAAT CJ, SILLEVIS SMITT PA, VECHT CJ, VAN DEN BENT MJ. Leptomeningeal metastasis after surgical resection of brain metastases. J Neurol Neurosurg Psychiatry 1999;66:225-7. 